\title{
«Mediziner können nicht alles alleine machen»
}

\section{Daniel Lüthi}

Freier Journalist und Fotograf, Medientrainer, Bern

Sicher liegt es auch an der Jahreszeit, dass der Gedanke an den Übergang und ans Ende so nahe ist. Ein Jahreswechsel kann nachdenklich stimmen. Das Sinnieren über Anfang und Ende hat ganz stark aber auch mit der Arbeit dieser Frau zu tun: Geburt und Tod, Leben und Sterben sind in ihrem Alltag eng verbunden miteinander. Beginnen wir mit dem Schluss dieser Begegnung. Bei Wind, Regen und Kälte stehen wir hinter dem Spital im nassen Rasen, zwischen verschieden hohen Granitstelen. "Hier nehmen wir in Würde von unseren frühverlorenen Kindern Abschied», sagt Karin Klemm, und es tönt ganz natürlich.

\section{Ein Ritual - auch für Mitarbeitende}

Viermal pro Jahr gebe es hier eine Zeremonie für jeweils etwa zehn bis zwanzig kleine Geschöpfe, die im Mutterleib gestorben sind. Beispielsweise komme musizierend ein Panflötist über den grünen Hügel, erzählt Klemm, dann würden die Anwesenden die Asche der Kinder, die vor der 22. Woche gestorben sind, in den entsprechenden Behälter in der Mitte des Gemeinschaftsgrabs eingiessen. Ein Chefarzt habe an dieser Zeremonie auch schon teilgenommen. Und es sei schon vorgekommen, dass keine Angehörigen dabei

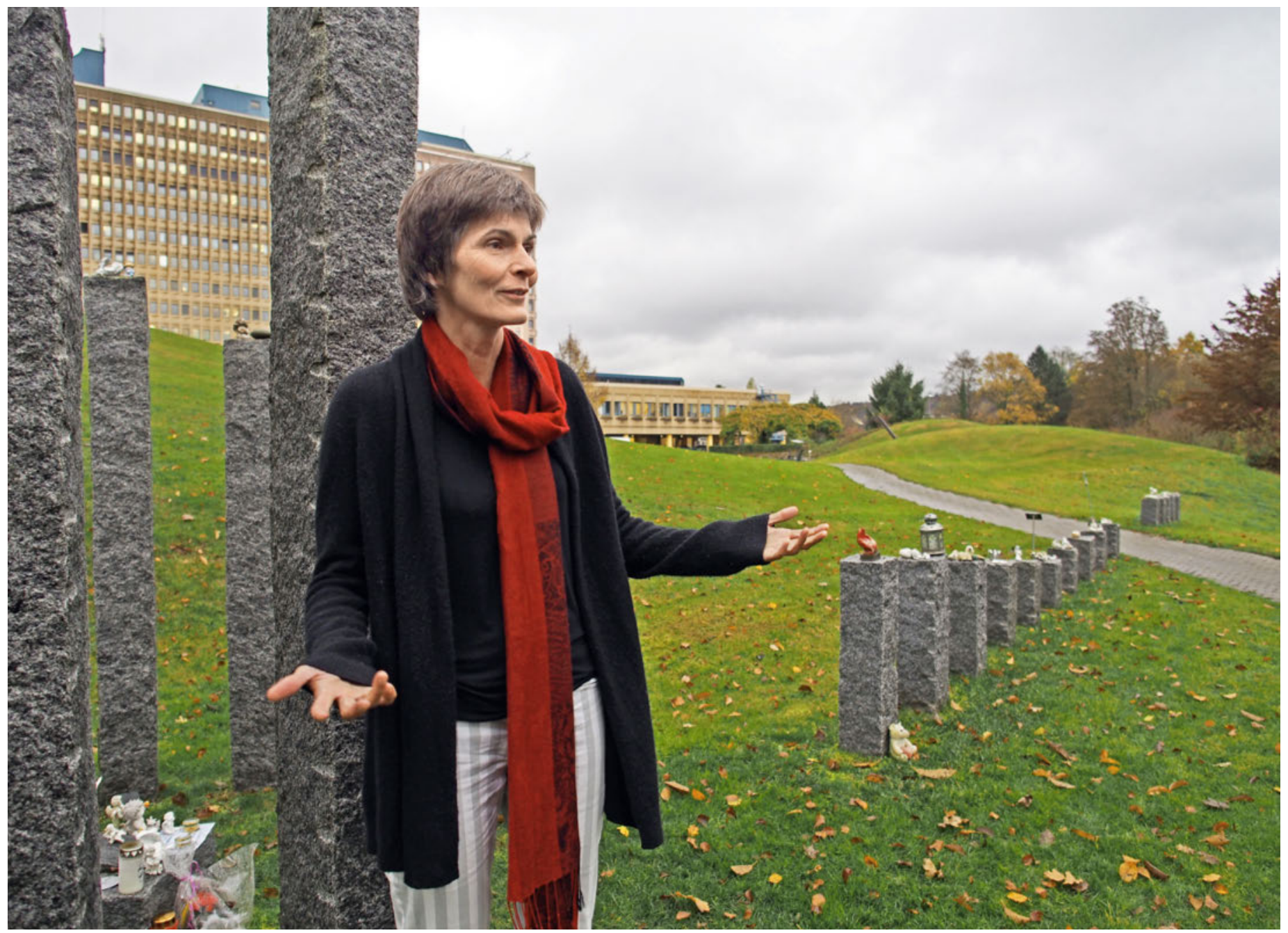


gewesen seien, dass sie mit dem Musiker, einer Hebamme und einer Pflegefachfrau alleine Abschied genommen habe. «Rituale, Seelsorge brauchen alle, auch Spitalangestellte.»

Eben habe sie das zehnjährige Jubiläum «ihrer» Gedenkstätte feiern können, berichtet mit spürbarem Stolz die grossgewachsene Frau, und nach wie vor sei «ihr» Spital das einzige in der Schweiz mit einem solchen Ort. Es geht eine spezielle Kraft von ihm aus, auch eine symbolische: Der Weg, der hierhin führt, unterbricht die Linie der Steinstelen dort, wo sie noch klein sind. Immer mehr ragen sie anschliessend in die Höhe, dem Himmel zu. Vorne der farbige Wald, im Hintergrund der riesige, hellerleuchtete Spitalbau mit seinem modernen Annex. Aus der Ruhe der Natur heraus lässt sich das dortige geschäftige Treiben nur noch erahnen.

\section{Ein Ort der Stille}

Auch im Innern des Spitals, diesem geschäftigen, oft hektischen Mikrokosmos, gibt es einen Ort der Stille. Er heisst "Andachtsraum», ist gross und für alle rund um die Uhr zugänglich. "Ab und zu erledigen hier Ärztinnen und Ärzte ein privates Telefon», berichtet Klemm amüsiert, jetzt gerade kommt ein Patient, seinen Infusionsständer vor sich herschiebend. Vorne in der Ecke entzündet er ein Kerzlein und betet. In der gegenüberliegenden Ecke liegt - nach Mekka ausgerichtet - ein kleiner Gebetsteppich, den Muslime offenbar regelmässig benutzen. Im Gästebuch machen Besucherinnen und Besucher ihre Hoffnungen und Wünsche öffentlich. «Lieber Gott», steht hier beispielsweise, «trage mich bitte in meiner schwierigen Lebenssituation.»

\section{«Ich rede nicht gerne formelhaft-religiös daher.»}

In der Mitte des Raums steht ein schlichter Altar aus Holz, hinten eine kleine Orgel. Der Blick geht durch die riesige Fensterfront ins Grüne, zu einem mächtigen Brunnen mit vielen Fontänen und weiter bis zur Gedenkstätte mit den Granitstelen und zum Wald. «Wir sind die Kirche im Spital», sagt Karin Klemm, «und 'Seelsorge' bedeutet für uns 'Einladung zur Begegnung'.» Und als wollte sie beweisen, dass Seelsorge keine abgehobene, esoterische Sache sein muss, wendet sie sich der kleinen Plastikflasche zu, die neben dem Altar steht. "Es ist wichtig, dass ich, nach dem Friedensgruss am Ende eines Gottesdienstes und bevor ich die Hostien verteile, jeweils meine Hände desinfiziere», stellt Klemm ganz profan fest. «Und wenn der Norovirus kommt, ist meine erste Amtshandlung, das Weihwasser zu entfernen.»

\section{Karin Klemm}

Karin Klemm wurde 1964 in Schwäbisch Hall in Süddeutschland geboren. Ihr Theologiestudium in Tübingen schloss sie 1991 als Diplom-Theologin ab. Danach arbeitete sie in der gleichen Stadt während sieben Monaten in der KlinikSeelsorge. 1991 bis 1995 war sie in Solothurn als Pastoralassistentin in Ausbildung angestellt. 1995 bis 1998 führte

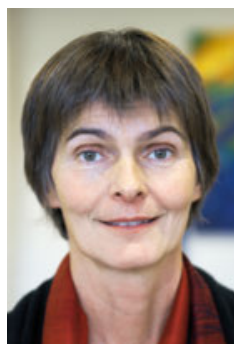

sie zusammen mit zwei Ordensfrauen im solothurnischen Flumenthal den Seelsorgeverband. Seit 1998 arbeitet sie als katholische Seelsorgerin am Kantonsspital Baden. Daneben engagiert sie sich in der Ausbildung von Seelsorgern und freiwilligen Sterbebegleiterinnen. Sie ist Präsidentin der Katholischen Spitalseelsorgevereinigung der Deutschschweiz. Karin Klemm ist verheiratet und Mutter eines 14-jährigen Sohnes. Sie lebt mit ihrer Familie in Dättwil, ganz in der Nähe ihres Arbeitsortes.

Nichts Pfarrherrliches haftet dieser Frau an, sie trägt Turnschuhe, und es wird sehr lange nicht einmal klar, ob sie die katholische oder die reformierte Glaubensrichtung vertritt. "Auf die Kirche als hierarchisch strukturierten, bürokratischen Laden hatte ich nie Lust», sagt sie, "ich rede auch nicht gerne formelhaftreligiös daher. Alles, was mit leeren Hülsen zu tun hat, macht mir Mühe.» Dies sei auch der Hauptgrund gewesen, warum es sie damals in die in dieser Hinsicht liberalere Schweiz gezogen habe. «Marien-Frömmigkeit ging übrigens auch an mir vorbei. Maria lernte ich erst in der feministischen Theologie kennen und schätzen.»

\section{Respekt, Vernetzung und der gute Geist}

Seit 18 Jahren arbeitet Karin Klemm in diesem Spital. Sie habe in dieser Zeit auch erlebt, wie sich die Spitalseelsorge verändert habe, erzählt sie. "Insgesamt sind wir multidisziplinärer und die Berührungsängste von Ärztinnen und Ärzten kleiner geworden. Das heisst allerdings nicht bloss, dass die klugen 'Halbgötter in Weiss' heute die Gnade haben, jemanden aus einer anderen Berufsgruppe anzuhören. Wir haben auch eine Bringschuld. Offenheit auf beiden Seiten ist gefragt.» Wobei mit Offenheit unter anderem Vernetzung gemeint ist, und dies nicht nur in der letzten Phase eines Patientenlebens und nicht nur mit Seelsorgerinnen und Seelsorgern. Karin Klemm sagt es so: «Mediziner können nicht alles alleine machen - dies haben die meisten von ihnen auch verstanden.» Mangelnden Respekt vor anderen Fachleuten erlebe sie kaum. «Ein herablassendes, abweisendes Verhalten seitens der Ärztinnen und Ärzte gab es früher selten, heute kaum 
mehr.» Natürlich erlebt auch sie, wie viele hier unter zunehmendem Kosten- und Zeitdruck leiden. «Im Spannungsfeld zwischen Geld und Geist will der gute Geist gepflegt und gehütet werden", kommentiert sie. Gerade in dieser speziellen Zeit, während der Festtage, komme es - beispielsweise in einem Lift an einem Sonn- oder Feiertag - zu speziellen interdisziplinären Begegnungen. «Ah ja, genau, du hast ja auch unregelmässige Arbeitszeiten und Pikettdienste», habe ein Arzt, etwas überrascht, bei einer solchen Gelegenheit einmal zu ihr gesagt.

Was macht sie denn konkret? Als Frau darf sie ja keine Sakramente spenden. Zum Beispiel: zuhören, da sein. Dabei sein, wenn jemand ein Untersuchungsergebnis erwartet. «Nach den Ressourcen fragen. Oder im Gebär-

\section{"Offenheit auf beiden Seiten ist gefragt.»}

saal das Entsetzen mitaushalten, wenn eine Frau ihr Kind verloren hat.» Oder, wenn dies gewünscht wird, ein Ritual mit Salböl feiern. "Das hilft, zur Ruhe zu kommen», erklärt Klemm. Sie bemerkt die fragenden Augen ihres Gegenübers und holt, zwecks Demonstration, in der Sakristei auf der anderen Seite des Spitalflurs das entsprechende kleine Metallgefäss. Es enthält eine Watte, die mit wohlriechendem Öl getränkt ist. «Ein sinnlicher Akt», kommentiert Klemm, während sie mit dem Mittelfinger das Öl in sanft kreisenden Bewegungen auf dem Handrücken einmassiert. «Wenn das jemand will, bedeutet es meistens, dass etwas zu Ende geht. Nicht immer ist es das Leben. Manchmal zum Beispiel die Hoffnung, wieder fit zu werden. Das Eingeständnis, gebrechlicher zu sein, als man es bisher wahrhaben wollte, ist schon ein grosser Schritt.»

\section{Eine persönliche Weihnachtsgeschichte}

Die Frage, was ihr Weihnachten bedeute, beantwortet Karin Klemm mit einer persönlichen Geschichte. Sie erinnert sich an eine spezielle Liturgie an einem Heiligabend, hier in diesem Raum. Viele Leute - auch von aussen - waren versammelt, eine junge Frau, die wegen eines Unterleibtumors am gleichen Tag operiert worden war, wurde im Bett hineingestossen. «Und das an diesem Geburtsfest», fügt Theologin Klemm hinzu. «Ich erzählte die Weihnachtsgeschichte. Nämlich, wie eine Frau unter widrigsten Umständen ihren Sohn auf die Welt bringen musste - in einem Stall bei den Tieren, ohne Hebamme oder andere unterstützende Frauen. Das Leben sucht sich seinen Weg, sagte ich, auch unter widrigsten Umständen.» Dann hätten die Anwesenden Kerzen angezündet und an einen für sie bedeutungsvollen Ort gestellt. «Die Frau im Bett hat mich gebeten, ihre Kerze an den Ort zu stellen, den wir mit 'neues Leben' betitelt hatten. Ich musste kämpfen, dass ich in meiner Rührung nicht unterging.» Auch das gehört zu dieser Seelsorgerin: dass sie sich selber vom Bedürfnis nach Seelsorge nicht ausnimmt. Einmal habe sie während Monaten eine junge Frau betreut, die in einem Autounfall ihren Mann und ihre beiden Kinder verloren hatte. "Am Schluss hat sie sich das Leben genommen, und ich musste sie beerdigen. Vorher aber hatte ich noch einen anderen Gottesdienst, und dort durfte ich von dieser Tragödie nichts sagen. Ich war am Ende.»

Wie kam und wie kommt sie jeweils wieder zu Kräften? «Professionelle Supervision ist obligatorisch», antwortet Klemm klar. Daneben nehme sie aber regelmässig auch die Unterstützung von anderen Fachfrauen in Anspruch, diejenige einer Psychologin und einer Traumatherapeutin beispielsweise.

Gerne jogge sie im Wald oder wandere in den Bergen. Und: «Wenn immer möglich, reise ich einmal pro Jahr ans Meer. Ich liebe es, im Meer zu baden und zu schwimmen. Ich lasse mich gerne tragen.»

Bildnachweis

Fotos Daniel Lüthi 